\title{
Photon sidebands of the ground state and the excited state of a quantum dot: A nonequilibrium Green-function approach
}

\author{
Qing-feng Sun \\ Department of Physics, The University of Hong Kong, Pokfulam Road, Hong Kong, China \\ and Department of Physics, Peking University, Beijing 100871, China \\ Jian Wang \\ Department of Physics, The University of Hong Kong, Pokfulam Road, Hong Kong, China \\ Tsung-han Lin ${ }^{*}$ \\ Department of Physics, The University of Hong Kong, Pokfulam Road, Hong Kong, China \\ and Department of Physics, Peking University, Beijing 100871, China
}

(Received 26 June 1998)

\begin{abstract}
The electron tunneling through an ultrasmall quantum dot in the presence of time-dependent microwave (MW) fields is studied. In the investigation, two single electronic states (the ground state and the excited state) and the intradot Coulomb interaction are considered. Assuming the tunneling through the system as a coherent process, the time-dependent current and the average current are derived using the nonequilibrium Greenfunction method. Then we consider two special cases with $\hbar \omega>\Delta \epsilon$ and $\hbar \omega<\Delta \epsilon$, respectively, where $\omega$ is the frequency of MW fields and $\Delta \epsilon$ is the energy difference between two electronic states. Both the sidebands of the photon-assisted tunneling originated from the ground state, and, in particular, from the excited state are obtained, which is in good agreement with the recent experiment by Oosterkamp et al. [Phys. Rev. Lett. 78, 1536 (1997)]. Moreover, the dependence of the integrated average current on the intensity of MW fields is also discussed, and attributed to the many-body effect of the quantum dot. [S0163-1829(98)01543-4]
\end{abstract}

\section{INTRODUCTION}

The electron tunneling through a mesoscopic system in the presence of time-varying microwave (MW) fields has been attracting more and more attention over the past few years. An essential feature is that the electron tunneling through the system can exchange the energy of $n \hbar \omega$ with MW fields $(n= \pm 1, \pm 2, \ldots, \omega$ is the frequency of MW fields), leading to the opening of new inelastic tunneling channels. This phenomenon has been well known as the photon-assisted tunneling (PAT). Back in the early 1960s, in the pioneering work by Tien and Gordon, the PAT through superconductor-insulator-superconductor films has been studied. ${ }^{1,2}$ In the last decade, the PAT through various nanostructures were extensively investigated.

Theoretically, Wingreen and co-workers presented a general formalism for the time-dependent coherent transport by the nonequilibrium Green-function (NEGF) method under the adiabatic approximation. ${ }^{3,4}$ Inarrea and co-workers dealt with the external electromagnetic field by the secondquantization method. ${ }^{5,6}$ Yakubo, Feng, and $\mathrm{Hu}$ investigated the condition for the strong influence of the PAT. ${ }^{7}$ Bruder and Schoeller considered the tunneling as a sequential process and used the nonMarkovian master equation to investigate the tunneling through a quantum dot with intradot Coulomb interaction. ${ }^{8}$ Oosterkamp et al. studied PAT through a quantum dot with multiple states and the intradot Coulomb interaction by using the master equation. ${ }^{9}$ Furthermore, the quenching or lack of quenching of the photon sidebands, ${ }^{10-12}$ the photon-electron pumping effect, ${ }^{13-15}$ the tunneling through a quantum well with transitions between the in- trawell levels, ${ }^{16}$ and the time-dependent dissipative transport $^{17}$ have also been investigated. Very recently, Pedersen and Büttiker have investigated the PAT using the scattering-matrix approach where the internal potential and the displacement current are considered. ${ }^{18}$

Experimentally, the observations of PAT have been reported in systems of superlattices, ${ }^{19,20}$ the quantum dot, ${ }^{21}$ and double quantum dots, ${ }^{22}$ etc. The photon-electron pump in a quantum dot driven by an asymmetric MW field has been observed by Kouwenhoven et al. ${ }^{15,23}$

Recently, Oosterkamp et al. ${ }^{24}$ investigated the PAT through an ultrasmall quantum dot in which the energy difference between the ground state and the first excited state, $\Delta \epsilon$, is larger than both the thermal energy $k_{B} \mathcal{T}$ and the linewidths $\Gamma$. For photon energy $\hbar \omega<\Delta \epsilon$, they found photon sideband resonances originated from the ground state, while for $h \omega>\Delta \epsilon$, they observed the photon-induced excited state resonances. By assuming sequential tunneling of a single electron they also presented a theoretical explanation using the master equation approach. ${ }^{24}$ Later, Brune, Bruder, and Schoeller studied the PAT through a single interacting quantum dot with arbitrary number of discrete levels. ${ }^{25}$ By introducing a generalized rotating-wave approximation and taking into account transitions between discrete levels of the dot, they found satisfactory agreement between their result and the experiment by Oosterkamp et al. ${ }^{24}$

However, there are still two problems puzzling us. First, the side-band resonance at $\epsilon_{0}+\hbar \omega$ from the ground state has not been found in the experiment by Oosterkamp et al. ${ }^{24}$ but it does emerge according to the existing theory. ${ }^{8,9}$ Second, we noticed that in all experiments involving tunneling 
through the quantum dot, ${ }^{15,21-24}$ the integration $\int\left\langle I\left(v_{g}\right)\right\rangle d v_{g}$ obviously increases with the intensity of MW fields from experimental data, where $\left\langle I\left(v_{g}\right)\right\rangle$ is the average current through the quantum dot and $v_{g}$ is the gate voltage. However, it is easy to prove theoretically that the integration $\int\left\langle I\left(v_{g}\right)\right\rangle d v_{g}$ is a constant, independent of MW fields, as long as the Coulomb interaction is neglected. Does this dependency of the integrated average current on the intensity of MW fields originate from the intradot Coulomb interaction?

Motivated by the above-mentioned problems, we investigate in this paper the PAT of electron tunneling through an ultrasmall quantum dot in the presence of time-dependent MW fields. To simplify the discussion, we consider only two single-electron states of the dot: the ground state $\epsilon_{0}$ and the first excited state $\epsilon_{1}$. The intradot electron-electron Coulomb interaction is also included.

In contrast to the theories by Bruder and Schoeller, ${ }^{8}$ Oosterkamp et al. and Brune, Bruder, and Schoeller, ${ }^{25}$ here we consider the electron tunneling through the quantum dot as a coherent process even in the presence of MW fields. It is appropriate if the temperature is low enough and MW fields are coherent. We also take the adiabatic approximation for the external MW field as is done in Refs. 3, 4, and 14, i.e., the external oscillating potential only causes a rigid shift in the single-electron energy spectrum but no transition between different electronic states takes place. By using the NEGF method, the time-dependent current $I(t)$ and the average current $\langle I\rangle$ are derived. We are interested in two special cases, corresponding to the high-frequency $(\hbar \omega>\Delta \epsilon)$ and the low-frequency $(\hbar \omega<\Delta \epsilon)$ MW fields, respectively. For $\hbar \omega>\Delta \epsilon$, we investigate three different ways of applying MW fields: the symmetric, the slightly asymmetric, and the completely asymmetric way. In the symmetric situation, all photon sidebands emerge, either originating from the ground state or from the excited state. However, in slightly asymmetric MW fields with suitable magnitudes, we find that the PAT peak at $\epsilon_{0}+\hbar \omega$ becomes negligibly small. For the completely asymmetric situation, the photon-electron pumping effect occurs. In the case of $\hbar \omega<\Delta \epsilon$, the photoninduced excited-state resonance and the sidebands of the excited state can not occur if the intensity of MW fields is weak, which is the case corresponding to the experiment by Oosterkamp et al. ${ }^{24}$ However, if the intensity of MW fields is strong enough, the PAT originated from the excited state will emerge. Finally, we study the dependence of the integration $\int\langle I\rangle d v_{g}$ on the intensity of MW fields, and find that this dependency can be attributed to the intradot electron-electron Coulomb interaction.

The outline of this paper is as follows. In Sec. II, the model is presented and the nonequilibrium Green-function method is used to derive the time-dependent current $I(t)$ and the average current $\langle I\rangle$. In Sec. III, we study the case of $\hbar \omega>\Delta \epsilon$. The case of $\hbar \omega<\Delta \epsilon$ is studied in Sec. IV. In Sec. $\mathrm{V}$, we investigate the dependency of the integration $\int\langle I\rangle d v_{g}$ on the intensity of MW fields. A brief summary is presented in Sec. VI.

\section{MODEL AND FORMULATION}

The system under consideration is described by the Hamiltonian $H=H_{0}+H_{1}$ :

$$
\begin{gathered}
H_{0}=\sum_{k \in L} \epsilon_{k}(t) a_{k}^{\dagger} a_{k}+\sum_{p \in R} \epsilon_{p}(t) b_{p}^{\dagger} b_{p}+\sum_{j=0,1} \epsilon_{j}(t) c_{j}^{\dagger} c_{j} \\
+U c_{0}^{\dagger} c_{0} c_{1}^{\dagger} c_{1}, \\
H_{1}=\sum_{k, j} v_{k j} a_{k}^{\dagger} c_{j}+\sum_{p, j} v_{p j} b_{p}^{\dagger} c_{j}+\text { H.c., }
\end{gathered}
$$

where $a_{k}^{\dagger}\left(a_{k}\right), b_{p}^{\dagger}\left(b_{p}\right)$, and $c_{j}^{\dagger}\left(c_{j}\right)$ are the creation (annihilation) operators of the electronic states in the left lead, the right lead, and the dot, respectively. Here we assume that there are only two states in the quantum dot, i.e., the ground state $\epsilon_{0}$ and the first excited state $\epsilon_{1}$. We also take into account the intradot electron-electron Coulomb interaction (the $U$ term). As for the time-varying MW fields, we take the adiabatic approximation ${ }^{3,4}$ in which MW fields can be described by an oscillating potential and it only causes the single-electron energy spectrum a rigid shift: $\epsilon_{\alpha}(t)=\epsilon_{\alpha}$ $+\Delta_{\beta}(t)$, where $\beta=0, L$, and $R$ denotes the dot, the left lead, and the right lead, respectively, $\epsilon_{\alpha}$ is the time-independent single electron energy without MW fields, $\Delta_{\beta}(t)$ is the timedependent MW field with $\Delta_{\beta}(t)=\Delta_{\beta} \cos \omega t . \quad H_{1}$ denotes the tunneling part that is time independent.

In the following, we derive the general formulas of the time-dependent particle current $I(t)$ and the average current $\langle I\rangle$ by using the NEGF technique. ${ }^{3,4,14}$ The time-dependent current from the left lead to the quantum dot can be calculated from the evolution of the total number operator of the electrons in the left lead, $N_{L}=\Sigma_{k} a_{k}^{\dagger} a_{k}$, and one finds (in units of $\hbar=1$ )

$$
I_{L}(t)=-e\left\langle\dot{N}_{L}\right\rangle=i e\left\langle\left[N_{L}, H(t)\right]\right\rangle=2 e \operatorname{Re} \sum_{k, j} v_{k j} G_{j k}^{<}(t, t) .
$$

Here we define the Green function $G_{j k}^{<}\left(t, t^{\prime}\right)$ $\equiv i\left\langle a_{k}^{\dagger}\left(t^{\prime}\right) c_{j}(t)\right\rangle$. With the help of the Dyson equation, the Green function $G_{j k}^{<}\left(t, t^{\prime}\right)$ can be obtained from $G_{j j}^{<}\left(t, t^{\prime}\right)$ and $G_{j j}^{r}\left(t, t^{\prime}\right)$, where $G_{j j .}^{<}\left(t, t^{\prime}\right) \equiv i\left\langle c_{j}^{\dagger}\left(t^{\prime}\right) c_{j}(t)\right\rangle$ and $G_{j j}^{r}\left(t, t^{\prime}\right) \equiv$ $-i \theta\left(t-t^{\prime}\right)\left\langle\left\{c_{j}(t), c_{j}^{\dagger}\left(t^{\prime}\right)\right\}\right\rangle$. Then the time-dependent current $I_{L}(t)$ becomes $3,4,14$

$$
\begin{aligned}
I_{L}(t)= & -2 e \operatorname{Im} \int_{-\infty}^{t} d t_{1} \int \frac{d \epsilon}{2 \pi} \sum_{j} e^{-i \epsilon\left(t_{1}-t\right)} \\
& \times \exp \left(-i \int_{t}^{t_{1}} \Delta_{L}(\tau) d \tau\right) \Gamma_{j}^{L}(\epsilon)\left[G_{j j}^{<}\left(t, t_{1}\right)\right. \\
& \left.+f_{L}(\epsilon) G_{j j}^{r}\left(t, t_{1}\right)\right],
\end{aligned}
$$

in which $f_{\alpha}(\epsilon)=f\left(\epsilon-e V_{\alpha}\right)$ with $\alpha=L, R$ is the Fermi distribution function of electrons in the leads, $V_{\alpha}$ is the dc bias, and $\Gamma_{j}^{L}(\boldsymbol{\epsilon}) \equiv 2 \pi \Sigma_{k} v_{k j} v_{k j}^{*} \delta\left(\epsilon-\epsilon_{k}\right)=2 \pi \rho_{L}(\epsilon) v_{j}(\epsilon) v_{j}^{*}(\boldsymbol{\epsilon})$ is the generalized linewidth function, $\rho_{L}(\epsilon)$ is the density of states in the left lead. Using the Keldysh equation, $G_{j j}^{<}\left(t, t^{\prime}\right)$ is related to the retarted Green function $G_{j j}^{r}\left(t, t^{\prime}\right)$ as

$$
G_{j j}^{<}\left(t, t^{\prime}\right)=\iint d t_{1} d t_{2} G_{j j}^{r}\left(t, t_{1}\right) \Sigma_{j j}^{<}\left(t_{1}, t_{2}\right) G_{j j}^{a}\left(t_{2}, t^{\prime}\right),
$$

and the self-energy function $\Sigma_{j j}^{<}\left(t_{1}, t_{2}\right)$ is 


$$
\begin{aligned}
\Sigma_{j j}^{<}\left(t_{1}, t_{2}\right)= & i \int \frac{d \epsilon}{2 \pi} e^{-i \epsilon\left(t_{1}-t_{2}\right)} \sum_{\alpha \in L, R} f_{\alpha}(\epsilon) \Gamma_{j}^{\alpha} \\
& \times \exp \left(-i \int_{t_{2}}^{t_{1}} \Delta_{\alpha}(\tau) d \tau\right) .
\end{aligned}
$$

It is useful to introduce $A_{j}^{\alpha}(\epsilon, t)$ (where $\left.\alpha=L, R\right)$,

$$
\begin{aligned}
A_{j}^{\alpha}(\epsilon, t)= & \int_{-\infty}^{t} d t_{1} G_{j j}^{r}\left(t, t_{1}\right) \exp \left(-i \epsilon\left(t_{1}-t\right)\right. \\
& \left.-i \int_{t}^{t_{1}} d \tau \Delta_{\alpha}(\tau)\right) .
\end{aligned}
$$

Under the wide bandwidth approximation, ${ }^{26}$ i.e., the linewidth $\Gamma_{j}^{L(R)}(\epsilon)$ is independent of the energy, the Green function $G_{j j}^{<}(t, t)$ can be expressed in terms of $A_{j}^{\alpha}(\epsilon, t)$,

$$
G_{j j}^{<}(t, t)=i \int \frac{d \epsilon}{2 \pi} \sum_{\alpha \in L, R} f_{\alpha}(\epsilon) \Gamma_{j}^{\alpha}\left|A_{j}^{\alpha}(\epsilon, t)\right|^{2},
$$

and the time-dependent current $I_{L}(t)$ reduces to

$$
\begin{aligned}
I_{L}(t)= & -2 e \operatorname{Im} \sum_{j} \int \frac{d \epsilon}{2 \pi} f_{L}(\epsilon) \Gamma_{j}^{L} A_{j}^{L}(\epsilon, t) \\
& -e \operatorname{Im} \sum_{j} \Gamma_{j}^{L} G_{j j}^{<}(t, t) \\
= & -e \sum_{j} I_{j}^{L} \int \frac{d \epsilon}{2 \pi}\left\{\sum_{\alpha=L, R} f_{\alpha}(\epsilon) \Gamma_{j}^{\alpha}\left|A_{j}^{\alpha}(\epsilon, t)\right|^{2}\right. \\
& \left.+2 f_{L}(\epsilon) \operatorname{Im} A_{j}^{L}(\epsilon, t)\right\} .
\end{aligned}
$$

Since $\langle I\rangle \equiv\left\langle I_{L}(t)\right\rangle=-\left\langle I_{R}(t)\right\rangle$, the average current $\langle I\rangle$ is

$$
\begin{aligned}
\langle I\rangle= & -2 e \sum_{j} \frac{\Gamma_{j}^{L} \Gamma_{j}^{R}}{\Gamma_{j}^{L}+\Gamma_{j}^{R}} \int \frac{d \epsilon}{2 \pi}\left[f_{L}(\epsilon) \operatorname{Im}\left\langle A_{j}^{L}(\epsilon, t)\right\rangle\right. \\
& \left.-f_{R}(\epsilon) \operatorname{Im}\left\langle A_{j}^{R}(\epsilon, t)\right\rangle\right],
\end{aligned}
$$

where the time average is defined as

$$
\langle F(t)\rangle \equiv \lim _{T \rightarrow \infty} \int_{-T / 2}^{T / 2} F(t) d t .
$$

In the following, we will calculate $G_{j j}^{r}\left(t, t^{\prime}\right)$ and hence $A_{j}^{\alpha}(\epsilon, t)$. In order to calculate $G_{j j}^{r}\left(t, t^{\prime}\right)$, we first calculate the retarted Green function of the isolated dot $g_{j j}^{r}\left(t, t^{\prime}\right)$. By using the equation of motion (EOM), one easily finds

$$
\begin{aligned}
& {\left[i \frac{\partial}{\partial t}-\epsilon_{j}(t)\right] g_{j j}^{r}\left(t, t^{\prime}\right)} \\
& \quad=\delta\left(t-t^{\prime}\right)-i \theta\left(t-t^{\prime}\right) U\left\langle\left\{c_{j}(t) c_{\bar{J}}^{\dagger}(t) c_{\bar{J}}(t), c_{j}^{\dagger}\left(t^{\prime}\right)\right\}\right\rangle,
\end{aligned}
$$

$$
\begin{aligned}
& {\left[i \frac{\partial}{\partial t}-\epsilon_{j}(t)-U\right]\left[-e \theta\left(t-t^{\prime}\right)\left\langle\left\{c_{j}(t) c_{\bar{J}}^{\dagger}(t) c_{\bar{J}}(t), c_{j}^{\dagger}\left(t^{\prime}\right)\right\}\right\rangle\right]} \\
& \quad=\delta\left(t-t^{\prime}\right) n_{\bar{J}}(t)
\end{aligned}
$$

where $n_{\bar{J}}(t)$ is the occupation number of the state $\bar{j}$. Here, $j=0$ or 1 and $\bar{j}=1-j$. From Eqs. (11) and (12), $g_{j j}^{r}\left(t, t^{\prime}\right)$ can be obtained exactly as

$$
g_{j j}^{r}\left(t, t^{\prime}\right)=\left[1-n_{\bar{J}}\left(t^{\prime}\right)\right] g_{\epsilon_{j}}^{r}\left(t, t^{\prime}\right)+n_{\bar{J}}\left(t^{\prime}\right) g_{\epsilon_{j}+U}^{r}\left(t, t^{\prime}\right),
$$

where

$$
g_{\epsilon_{j}}^{r}\left(t, t^{\prime}\right) \equiv-i \theta\left(t-t^{\prime}\right) \exp \left(-i \int_{t^{\prime}}^{t} \epsilon_{j}(\tau) d \tau\right)
$$

and

$$
g_{\epsilon_{j}+U}^{r}\left(t, t^{\prime}\right) \equiv-i \theta\left(t-t^{\prime}\right) \exp \left(-i \int_{t^{\prime}}^{t}\left[\epsilon_{j}(\tau)+U\right] d \tau\right) \text {. }
$$

Next, we have to solve the Green function $G_{j j}^{r}\left(t, t^{\prime}\right)$. Using the equation of motion, one has

$$
\begin{aligned}
& {\left[i \frac{\partial}{\partial t}-\epsilon_{j}(t)\right] G_{j j}^{r}\left(t, t^{\prime}\right)} \\
& =\delta\left(t-t^{\prime}\right)-i \theta\left(t-t^{\prime}\right) U\left\langle\left\{c_{j}(t) c_{\bar{J}}^{\dagger}(t) c_{\bar{J}}(t), c_{j}^{\dagger}\left(t^{\prime}\right)\right\}\right\rangle \\
& +\sum_{k} v_{k j}^{*} G_{k j}^{r}\left(t, t^{\prime}\right)+\sum_{p} v_{p j}^{*} G_{p j}^{r}\left(t, t^{\prime}\right) \\
& {\left[i \frac{\partial}{\partial t}-\epsilon_{j}(t)-U\right]\left[-i \theta\left(t-t^{\prime}\right)\left\langle\left\{c_{j}(t) c_{\bar{J}}^{\dagger}(t) c_{\bar{J}}(t), c_{j}^{\dagger}\left(t^{\prime}\right)\right\}\right\rangle\right]} \\
& =\delta\left(t-t^{\prime}\right) n_{\bar{J}}(t)-i \theta\left(t-t^{\prime}\right) \\
& \times\left\{\sum_{k} v_{k j}^{*}\left\langle\left\{a_{k}(t) c_{\bar{J}}^{\dagger}(t) c_{\bar{J}}(t), c_{j}^{\dagger}\left(t^{\prime}\right)\right\}\right\rangle\right. \\
& +\sum_{p} v_{p j}^{*}\left\langle\left\{b_{k}(t) c_{J}^{\dagger}(t) c_{\bar{J}}(t), c_{j}^{\dagger}\left(t^{\prime}\right)\right\}\right\rangle \\
& +\sum_{k} v_{k J}^{*}\left\langle\left\{c_{j}(t) c_{j}^{\dagger}(t) a_{k}(t), c_{j}^{\dagger}\left(t^{\prime}\right)\right\}\right\rangle \\
& +\sum_{p} v_{p \bar{J}}^{*}\left\langle\left\{c_{j}(t) c_{\bar{J}}^{\dagger}(t) a_{k}(t), c_{j}^{\dagger}\left(t^{\prime}\right)\right\}\right\rangle \\
& -\sum_{k} v_{k J}\left\langle\left\{c_{j}(t) a_{k}^{\dagger}(t) c_{\bar{J}}(t), c_{j}^{\dagger}\left(t^{\prime}\right)\right\}\right\rangle \\
& \left.-\sum_{p} v_{p \bar{J}}\left\langle\left\{c_{j}(t) a_{k}^{\dagger}(t) c_{\bar{J}}(t), c_{j}^{\dagger}\left(t^{\prime}\right)\right\}\right\rangle\right\}
\end{aligned}
$$

where the two new Green functions $G_{k j}^{r}\left(t, t^{\prime}\right)$ and $G_{p j}^{r}\left(t, t^{\prime}\right)$ in Eq. (16) are defined as $G_{k j}^{r}\left(t, t^{\prime}\right)$ $\equiv-i \theta\left(t-t^{\prime}\right)\left\langle\left\{a_{k}(t), c_{j}^{\dagger}\left(t^{\prime}\right)\right\}\right\rangle \quad$ and $\quad G_{p j}^{r}\left(t, t^{\prime}\right) \equiv-i \theta(t$ $\left.-t^{\prime}\right)\left\langle\left\{b_{p}(t), c_{j}^{\dagger}\left(t^{\prime}\right)\right\}\right\rangle$. To obtain a closed form of the EOM, the higher-order many-particle Green functions need to be decoupled. We make the following decoupling approximation: 


$$
\begin{gathered}
\left\langle\left\{X(t) c_{\bar{J}}^{\dagger}(t) c_{\bar{J}}(t), c_{j}^{\dagger}\left(t^{\prime}\right)\right\}\right\rangle \approx n_{\bar{J}}(t)\left\langle\left\{X(t), c_{j}^{\dagger}\left(t^{\prime}\right)\right\}\right\rangle, \\
\left\langle\left\{c_{j}(t) c_{\bar{J}}^{t}(t) X(t), c_{j}^{\dagger}\left(t^{\prime}\right)\right\}\right\rangle \approx 0, \\
\left\langle\left\{c_{j}(t) X^{\dagger}(t) c_{\bar{J}}(t), c_{j}^{\dagger}\left(t^{\prime}\right)\right\}\right\rangle \approx 0,
\end{gathered}
$$

where $X=a_{k}$ or $b_{p}$. In contrast to our previous work, ${ }^{14}$ here we have to take the decoupling approximation to higher order, which is necessary for investigating the photon-induced excited-state resonances. To our knowledge, no such higherorder cutoff approximation has been given for the timedependent problem before. In this decoupling approximation the state $j$ is considered as a superposition of two states: one state is at energy $\epsilon_{j}(t)$ with probability $1-n_{\bar{J}}(t)$ while the state $\bar{j}$ is empty; the other state is at energy $\epsilon_{j}(t)+U$ weighted by $n_{\bar{J}}$, while the state $\bar{j}$ is occupied. Under this decoupling approximation, Eq. (17) becomes

$$
\begin{aligned}
& {\left[i \frac{\partial}{\partial t}-\epsilon_{j}(t)-U\right]\left[-i \theta\left(t-t^{\prime}\right)\left\langle\left\{c_{j}(t) c_{\bar{J}}^{\dagger}(t) c_{\bar{J}}(t), c_{j}^{\dagger}\left(t^{\prime}\right)\right\}\right\rangle\right]} \\
& =\delta\left(t-t^{\prime}\right) n_{\bar{J}}(t)+n_{\bar{J}}(t) \sum_{k} v_{k j}^{*} G_{k j}^{r}\left(t, t^{\prime}\right) \\
& \quad+n_{\bar{J}}(t) \sum_{p} v_{p j}^{*} G_{p j}^{r}\left(t, t^{\prime}\right)
\end{aligned}
$$

The new Green functions $G_{k j}^{r}\left(t, t^{\prime}\right)$ and $G_{p j}^{r}\left(t, t^{\prime}\right)$ in Eqs. (16) and (19) can be obtained by the Dyson equation

$$
G_{k(p) j}^{r}\left(t, t^{\prime}\right)=\int d t_{1} v_{k(p) j} g_{k(p)}^{r}\left(t, t_{1}\right) G_{j j}^{r}\left(t_{1}, t^{\prime}\right),
$$

where $g_{k(p)}^{r}\left(t, t_{1}\right)=-i \theta\left(t-t_{1}\right) \exp \left\{-i \int_{t_{1}}^{t} \epsilon_{k(p)}(\tau) d \tau\right\}$ is the exact retarted Green function of the electron of the left (right) lead. Substituting Eq. (20) into Eqs. (16) and (19), denoting the retarted self-energy $\Sigma_{j j}^{L(R) r}\left(t_{1}, t_{2}\right)$ $=\Sigma_{k(p)} v_{k(p) j}^{*} v_{k(p) j} g_{k(p)}^{r}\left(t_{1}, t_{2}\right)$, and $\Sigma_{j j}^{r}\left(t_{1}, t_{2}\right)=\Sigma_{j j}^{L r}\left(t_{1}, t_{2}\right)$ $+\sum_{j j}^{R r}\left(t_{1}, t_{2}\right)$, and with the help of $g_{\epsilon_{j}}^{r}$ and $g_{\epsilon_{j}+U}^{r}$, differential equations Eqs. (11) and (14) can be written as the following integral equations:

$$
\begin{aligned}
G_{j j}^{r}\left(t, t^{\prime}\right)= & g_{\epsilon_{j}}^{r}\left(t, t^{\prime}\right)+\iint d t_{1} d t_{2} g_{\epsilon_{j}}^{r}\left(t, t_{1}\right) \\
& \times \Sigma_{j j}^{r}\left(t_{1}, t_{2}\right) G_{j j}^{r}\left(t_{2}, t^{\prime}\right)+U \int d t_{1} g_{\epsilon_{j}}^{r}\left(t, t_{1}\right) \\
& \times\left[-i \theta\left(t_{1}-t^{\prime}\right)\left\langle\left\{c_{j}\left(t_{1}\right) c_{\bar{J}}^{\dagger}\left(t_{1}\right) c_{\bar{J}}\left(t_{1}\right), c_{j}^{\dagger}\left(t^{\prime}\right)\right\}\right\rangle\right],
\end{aligned}
$$

$$
\begin{aligned}
-i \theta & \left(t_{1}-t^{\prime}\right)\left\langle\left\{c_{j}(t) c_{\bar{J}}^{\dagger}(t) c_{\bar{J}}(t), c_{j}^{\dagger}\left(t^{\prime}\right)\right\}\right\rangle \\
= & n_{\bar{J}}\left(t^{\prime}\right) g_{\epsilon_{j}+U}^{r}\left(t, t^{\prime}\right)+\iint d t_{1} d t_{2} n_{J}\left(t_{1}\right) g_{\epsilon_{j}+U}^{r}\left(t, t_{1}\right) \\
& \times \Sigma_{j j}^{r}\left(t_{1}, t_{2}\right) G_{j j}^{r}\left(t_{2}, t^{\prime}\right)
\end{aligned}
$$

From Eqs. (22), (21), and (15), one easily finds

$$
\begin{aligned}
G_{j j}^{r}\left(t, t^{\prime}\right)= & g_{j j}^{r}\left(t, t^{\prime}\right)+\iint d t_{1} d t_{2} g_{j j}^{r}\left(t, t_{1}\right) \Sigma_{j j}^{r}\left(t_{1}, t_{2}\right) \\
& \times G_{j j}^{r}\left(t_{2}, t^{\prime}\right) .
\end{aligned}
$$

Under the wide bandwidth approximation, the retarted selfenergy $\Sigma_{j j}^{r}\left(t_{1}, t_{2}\right)$ reduces to

$$
\Sigma_{j j}^{r}\left(t_{1}, t_{2}\right)=-\frac{i}{2} \Gamma_{j} \delta\left(t_{1}-t_{2}\right),
$$

where $\Gamma_{j}=\Gamma_{j}^{L}+\Gamma_{j}^{R}$. In the following, we make the further simplifications: (1) $U$ is very large $(U \gg \Delta \epsilon)$. (2) $n_{\bar{J}}(t)$ is replaced by its average value $n_{\bar{J}}$. By iterating $G_{j j}^{r}\left(t, t^{\prime}\right)$ in Eq. (23), one obtains

$$
\begin{aligned}
G_{j j}^{r}\left(t, t^{\prime}\right)= & {\left[1-n_{\bar{J}}\right] g_{\epsilon_{j}}^{r}\left(t, t^{\prime}\right) e^{-\left(\Gamma_{j} / 2\right)\left(1-n_{\bar{J}}\right)\left(t-t^{\prime}\right)} } \\
& +n_{\bar{J}} g_{\epsilon_{j}+U}^{r}\left(t, t^{\prime}\right) e^{-\left(\Gamma_{j} / 2\right) n_{\bar{J}}\left(t-t^{\prime}\right)} .
\end{aligned}
$$

Obviously, $G_{j j}^{r}\left(t, t^{\prime}\right)$ has two resonances: one is at $\epsilon_{j}$ with the linewidth $\left(1-n_{\bar{J}}\right) \Gamma_{j}$ and the probability $\left(1-n_{\bar{J}}\right)$, while another state is empty; the other resonance is at $\epsilon_{j}+U$ with the linewidth $n_{\bar{J}} \Gamma_{j}$ and weighted by the probability $n_{\bar{J}}$, while another state is occupied. ${ }^{27}$ Substituting the expression of $G_{j j}^{r}\left(t, t_{1}\right)$ into Eq. (7), using $\epsilon_{\alpha}(t)=\epsilon_{\alpha}+\Delta_{\beta}(t)$ and $\Delta_{\beta}(t)$ $=\Delta_{\beta} \cos \omega t(\alpha=k, p, i$; and $\beta=L, R, 0)$, and carrying out the integration over $t_{1}$, then $A_{j}^{\alpha}(\epsilon, t)$ becomes

$$
\begin{aligned}
A_{j}^{\alpha}(\epsilon, t)= & \sum_{k, k^{\prime}} J_{k}\left(\frac{\Delta_{0}-\Delta_{\alpha}}{\omega}\right) J_{k^{\prime}}\left(\frac{\Delta_{\alpha}-\Delta_{0}}{\omega}\right) e^{i\left(k+k^{\prime}\right) \omega t} \\
& \times\left\{\frac{1-n_{\bar{J}}}{\epsilon-\epsilon_{j}-k^{\prime} \omega+i \frac{\Gamma_{j}\left(1-n_{\bar{J}}\right)}{2}}\right. \\
& \left.+\frac{n_{\bar{J}}}{\epsilon-\epsilon_{j}-U-k^{\prime} \omega+i \frac{\Gamma_{j} n_{\bar{J}}}{2}}\right\}
\end{aligned}
$$

Substituting $A_{j}^{\alpha}(\epsilon, t)$ into Eqs. (9) and (10), the timedependent current $I_{L}(t)$ and the average current $\langle I\rangle$ are obtained immediately. Notice that these formulas of the current satisfy the gauge invariance in the following sense: if the voltages of the left lead, the right lead, and the gate voltage $v_{g}$ (which controls the intradot electronic energy levels $\epsilon_{j}$ $=\epsilon_{j}^{0}+e v_{g}$ ) are shifted by the same amount, the current does not change. ${ }^{28}$ The current $I(t)$ can be separated into two parts $I_{0}(t)$ and $I_{1}(t)$, where $I_{j}(t)(j=0,1)$ is the current through the state $j$. The current formulas obtained in this paper should be applied to an ultrasmall quantum dot with $U \gg \Delta \epsilon$, but no restriction about the bias voltage and the intensity of MW fields. The average occupation number in the state $j, n_{j}$, should be calculated self-consistently:

$$
n_{j}=\left\langle\operatorname{Im} G_{j j}^{<}(t, t)\right\rangle=\int \frac{d \epsilon}{2 \pi} \sum_{\alpha \in L, R} f_{\alpha}(\epsilon) \Gamma^{\alpha}\left\langle\left|A_{j}^{\alpha}(\epsilon, t)\right|^{2}\right\rangle .
$$

In numerical studies, we take the following approximations: (1) $U=\infty$ since $U \approx 10 \Delta \epsilon$ in the experiment by 

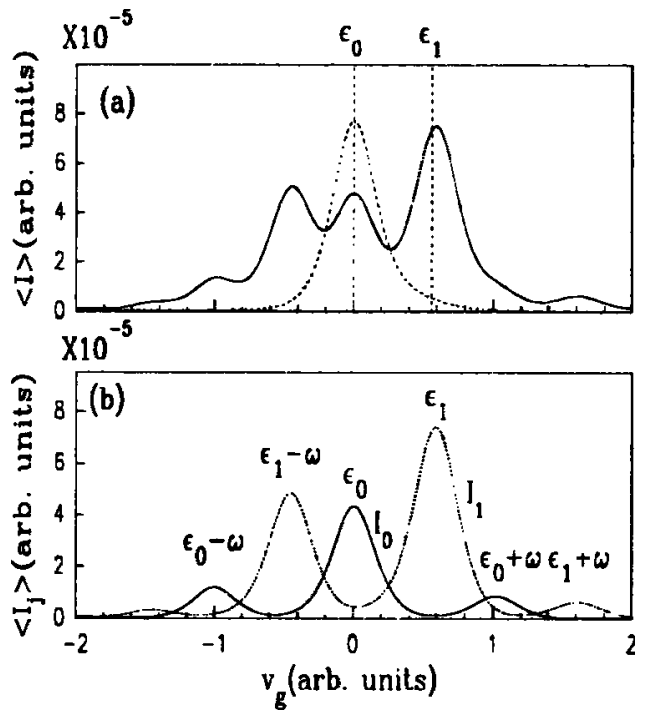

FIG. 1. (a) $\langle I\rangle$ vs $v_{g}$ for $\hbar \omega>\Delta \omega$ and the symmetric MW fields. The dotted curve corresponds to the case without MW fields. (b) $\left\langle I_{0}\right\rangle$ and $\left\langle I_{1}\right\rangle$ vs $v_{g}$, where $\Delta_{L}=\Delta_{R}=0.9, \Delta_{0}=0, \omega=1, \Delta \epsilon$ $=0.55, \Gamma_{0}=0.006, \Gamma_{1}=0.03$, the bias voltage $V=0.02$, and the temperature $\mathcal{T}=0.1$.

Oosterkamp et al. ${ }^{24}(2)$ the symmetric barriers $\left(\Gamma_{j}^{L}=\Gamma_{j}^{R}\right)$. In $U=\infty$ limit, the second term in the bracket of $A_{j}^{\alpha}(\epsilon, t)$, Eq. (26), becomes zero; then the average current $\langle I\rangle$ and the selfconsistent equation of the occupation number reduce to

$$
\begin{aligned}
\langle I\rangle= & e \sum_{j} \Gamma_{j}^{L} \Gamma_{j}^{R} \int \frac{d \epsilon}{2 \pi} \sum_{k}\left\{f_{L}(\epsilon) J_{k}^{2}\left(\frac{\Delta_{0}-\Delta_{L}}{\omega}\right)\right. \\
& \left.-f_{R}(\epsilon) J_{k}^{2}\left(\frac{\Delta_{0}-\Delta_{R}}{\omega}\right)\right\} \\
& \times \frac{\left(1-n_{\bar{J}}\right)^{2}}{\left(\epsilon-\epsilon_{j}+k \omega\right)^{2}+\left[\frac{\Gamma_{j}\left(1-n_{\bar{J}}\right)}{2}\right]^{2},} \\
n_{j}= & \int \frac{d \epsilon}{2 \pi} \sum_{\alpha \in L, R} f_{\alpha}(\epsilon) \Gamma^{\alpha} \sum_{k} J_{k}^{2}\left(\frac{\Delta_{0}-\Delta_{\alpha}}{\omega}\right) \\
& \times \mid \frac{1-n_{\bar{J}}}{\Gamma_{j}\left(1-n_{\bar{J}}\right)} \\
& \mid \epsilon-\epsilon_{j}+k \omega+i \frac{1}{2} \cdot
\end{aligned}
$$

\section{THE CASE OF $\hbar \omega>\Delta \epsilon$}

On the basis of the current formula Eq. (28), now we start to study the properties of the average current, for the case of $\hbar \omega>\Delta \epsilon$, in which the photon-induced excited-state resonance and its sidebands resonances will emerge even in weak MW fields, $\Delta_{\alpha} / \omega<1(\alpha=L, R, 0)$. In the following, we discuss three different situations of applied MW fields: the symmetric, the slightly asymmetric, and the completely asymmetric.

(a) The symmetric MW fields $\left(\Delta_{L}=\Delta_{R} \equiv \Delta, \Delta_{0}=0\right)$. In this case, MW fields are symmetrically applied on the left and the right leads. Figure 1(a) shows the average current $\langle I\rangle$ vs the gate voltage $v_{g}$ at small bias. One can clearly see

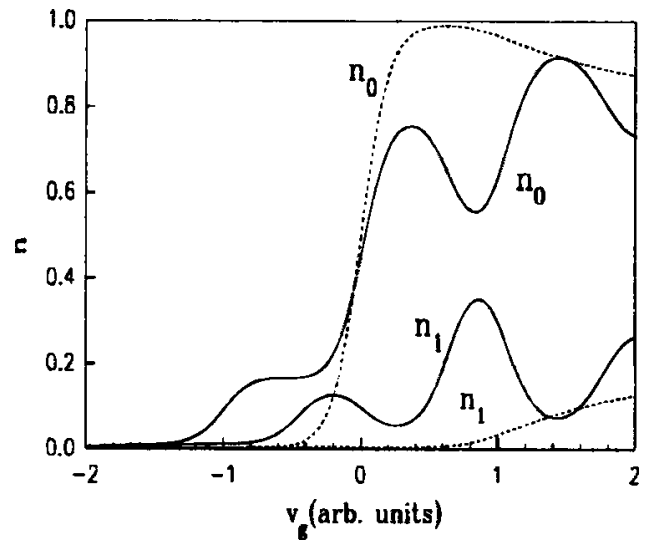

FIG. 2. $\left\langle n_{j}\right\rangle(j=0,1)$ vs $v_{g}$ with the same parameters as in Fig. 1. The dotted curves correspond to the case without MW fields.

peaks located not only at the ground state $\epsilon_{0}$ and its sidebands $\epsilon_{0} \pm n \hbar \omega(n=1,2, \ldots)$, but also at the first excited state $\epsilon_{1}$ and its sidebands $\epsilon_{1} \pm n \hbar 1 \omega(n=1,2, \ldots)$. Notice that the peak at $\epsilon_{0}+\hbar \omega$ is rather weak. Figure 1(b) shows the current components $\left\langle I_{0}\right\rangle$ and $\left\langle I_{1}\right\rangle$ corresponding to the part through the ground state and the first excited state, respectively. Without MW fields, $\left\langle I_{0}\right\rangle$ only has a single resonant peak at $\epsilon_{0}$ and $\left\langle I_{1}\right\rangle$ is almost zero. However, in the presence of MW fields, $\left\langle I_{0}\right\rangle$ will be split into a series of peaks, and $\left\langle I_{1}\right\rangle$ becomes much larger and exhibits sideband peaks, too. The sideband peaks of the ground state are slightly asymmetric, but for the first excited state they are heavily asymmetric. For example, the peak at $\epsilon_{1}-\hbar \omega$ is much higher than that at $\epsilon_{1}+\hbar \omega$ [see Fig. 1(b)]. This is because the height of the sideband peak at $\epsilon_{j} \pm n \hbar \omega(j$ $=0,1 ; n=0,1,2, \ldots)$ is directly proportional to (1 $\left.-n_{\bar{J}}\right) J_{n}^{2}(\Delta / \omega)$. With the increase of the gate voltage $v_{g}$, the occupation number $n_{0}$ varies significantly, almost changed from 0 to 1 , leading to heavily asymmetric sideband peaks for the first excited state. However, the occupation number $n_{1}$ only changes slightly, leading to slightly asymmetric sideband peaks for the ground state.

In the following, we study the behavior of the occupation number $n_{j}$. In the limit of $U=\infty$, based on Eq. (29) one can prove that the inequality $n_{0}+n_{1} \leqslant 1$ holds, which is independent of the magnitudes of MW fields and the gate voltage $v_{g}$. It means that electrons cannot occupy the ground state and the first excited state simultaneously. Without MW fields, the occupation number of the ground state $n_{0}$ is almost zero when $v_{g}<\epsilon_{0}$, increases suddenly like a "step" around $v_{g} \approx \epsilon_{0}$, and then slightly decreases for $v_{g}>\epsilon_{1}$ (see Fig. 2). The slight reduction of $n_{0}$ for $v_{g}>\epsilon_{1}$ is due to the fact that the excited state has moved near the Fermi levels of the leads, so it has a certain probability to be occupied due to the resonance. As a result, the electron-electron interaction between the ground state and the excited state leads to a slight reduction of $n_{0}$. In the presence of MW fields, the behavior of the occupation number $n_{0}$ vs the gate voltage $v_{g}$ changes: (1) The "step" in the curve of $n_{0}$ vs $v_{g}$ is split into a series of the sub-"step" at $\epsilon_{0} \pm n \hbar \omega$ due to the sideband splitting of the state $\epsilon_{0}$. (2) When $v_{g}$ pass through $\epsilon_{1}$ $\pm n \hbar \omega, n_{0}$ obviously reduces, because at this $v_{g}$ a sideband of the first excited state is pulled down below both of the 

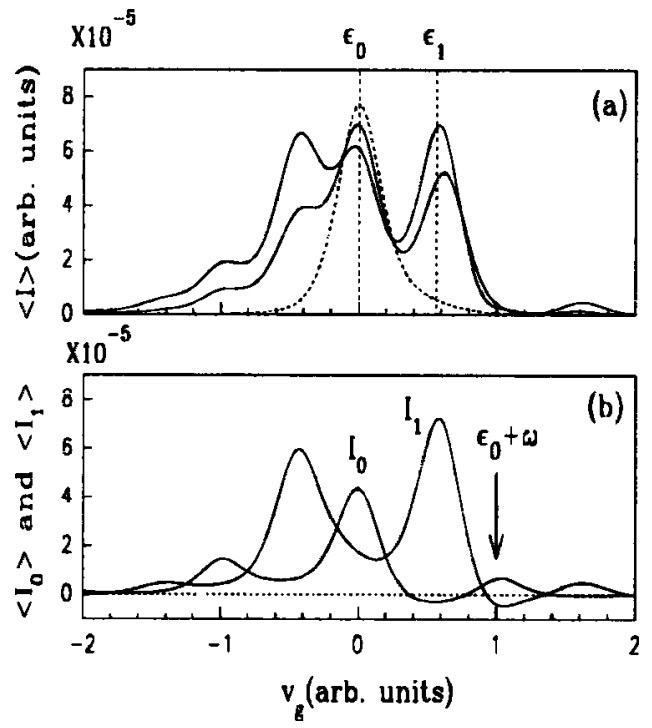

FIG. 3. (a) $\langle I\rangle$ vs $v_{g}$ for $\hbar \omega>\Delta \epsilon$ and slightly asymmetric MW fields. The two solid curves correspond to $\Delta_{L}=0.9, \Delta_{L}-\Delta_{R}$ $=0.01$, and $\Delta_{L}=0.6, \Delta_{L}-\Delta_{R}=0.007$, respectively. Other parameters are the same as in Fig. 1. The dotted curve corresponds to the case without MW fields. (b) $\left\langle I_{0}\right\rangle$ and $\left\langle I_{1}\right\rangle$ vs $v_{g}$ for $\Delta_{L}=0.9, \Delta_{L}$ $-\Delta_{R}=0.01$, and $\hbar \omega>\Delta \epsilon$.

Fermi levels of two leads, leading to an increase of $n_{1}$ and a reduction of $n_{0}$.

(b) The slightly asymmetric case. In the completely symmetric case, although the sideband peak at $\epsilon_{0}+\hbar \omega$ is rather small, it can still be seen. Instead, for slightly asymmetric MW fields with suitable magnitudes, the sideband peak $\epsilon_{0}$ $+\hbar \omega$ will disappear and the average current $\langle I\rangle$ is almost zero at that $v_{g}$, while all the other peaks still exist [see Fig. 3(a)]. This is well consistent with the experiment by Oosterkamp et al. ${ }^{24}$ The reason of the disappearance of the sideband peak at $\epsilon_{0}+\hbar \omega$ is that for the slightly asymmetric case, $\left\langle I_{0}\right\rangle$ still has a sideband peak at $\epsilon_{0}+\hbar \omega$, but $\left\langle I_{1}\right\rangle$ has a negative current at the same $v_{g}$ [see Fig. 3(b)]. These two opposite trends make the current $\langle I\rangle$ almost zero at $\epsilon_{0}$ $+\hbar \omega$. It should be pointed out that the asymmetry of MW fields needed for this disappearance is rather small. In fact for the chosen parameters, $\left(\Delta_{L}-\Delta_{R}\right) /\left(\Delta_{L}+\Delta_{R}\right)$ is about 0.005 . Naturally, for the slightly asymmetric external fields and at a certain gate voltage $v_{g}$, the average current $\langle I\rangle$ may also have either small negative or positive value, depending on which trend is stronger. Notice that for the completely symmetric case, the negative current will never emerge.

(c) The completely asymmetric case. In this case, MW fields are only applied on the left lead. Figure 4(a) shows the dependence of the current $\langle I\rangle$ on the gate voltage $v_{g}$. A shoulder emerges on the left-hand side, and a negative current (i.e., photon-electron pumping effect) emerges on the right-hand side of the resonant peak. Figure 4(b) presents the differential conductance $d\langle I\rangle / d v_{g}$ vs the gate voltage $v_{g}$. One can clearly see the PAT peaks from the ground state at $\epsilon_{0} \pm \hbar \omega$ and from the excited state at $\epsilon_{1} \pm \hbar \omega$. The sideband peak at $\epsilon_{0}-\hbar \omega$ is slightly higher than that at $\epsilon_{0}+\hbar \omega$. However, the PAT sideband peak at $\epsilon_{1}+\hbar \omega$ is very weak, since the height of the sideband peak is proportion to $1-n_{0}$, and the occupation number $n_{0}$ is almost one at $v_{g}=\epsilon_{1}+\hbar \omega$. It is

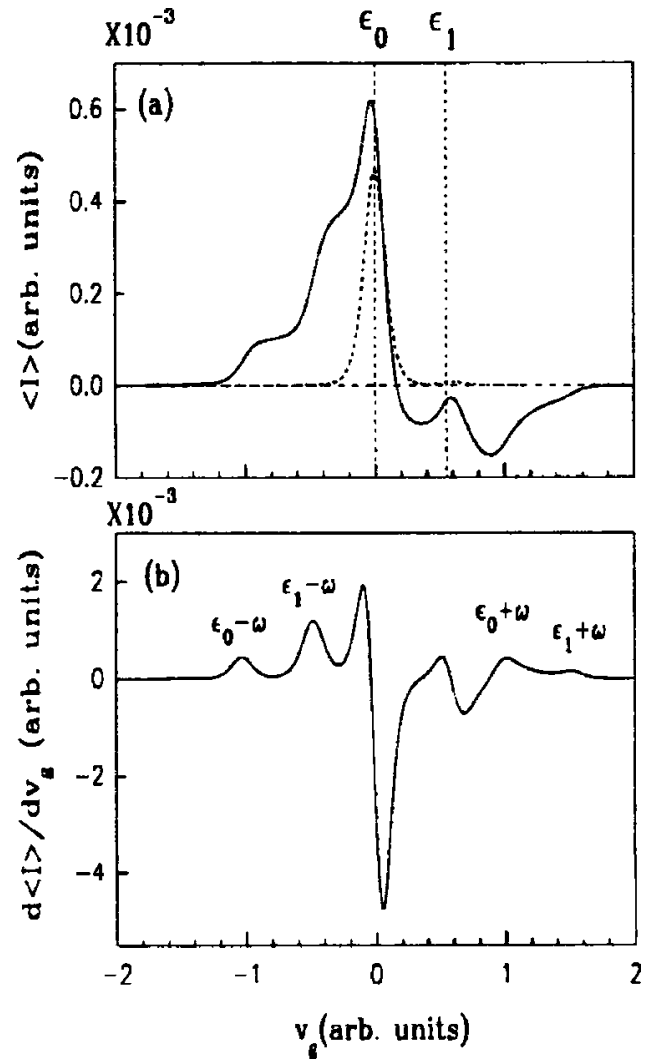

FIG. 4. (a) $\langle I\rangle$ vs $v_{g}$ for $\hbar \omega>\Delta \epsilon$ and completely asymmetric MW fields. The dotted curve corresponds to the case without MW field. (b) $d\langle I\rangle / d v_{g}$ vs $v_{g}$, where $\Delta_{L}=0.4, \Delta_{R}=\Delta_{0}=0, \omega=1, \Delta \epsilon$ $=0.55, \Gamma_{0}=0.01, \Gamma_{1}=0.03, V=0.04$, and $\mathcal{T}=0.05$.

worth mentioning that the occupation number $n_{0}$ is much closer to unity when the MW field is only applied on the left lead than applied on both leads. Therefore, the sideband peak at $\epsilon_{1}+\hbar \omega$ is always weaker for the asymmetric case than for the symmetric case.

\section{THE CASE OF $\hbar \omega<\Delta \epsilon$}

For the case of $\hbar \omega<\Delta \epsilon$, if the intensity of MW fields are weak $\left(\Delta_{\alpha} / \omega<1, \alpha=L, R, 0\right)$, the excited state does not participate the PAT process, only the resonances of the ground state and its sideband emerge. This result is similar to the case of single-level dot. ${ }^{29}$ The slight asymmetry of the sideband peaks as mentioned in Ref. 24 is due to the fact that the occupation number $n_{1}$ of the excited state slightly changes with the gate voltage $v_{g}$.

However, if the intensity of MW fields is strong $\left(\Delta_{\alpha} / \omega\right.$ $>1$ ), the resonance of the first excited state can still be induced. Figure 5(a) shows the current $\langle I\rangle$ vs $v_{g}$ for the case with strong fields. One finds not only peaks located at $\epsilon_{0}$ $\pm n \hbar \omega(n=1,2, \ldots)$ but peaks located at $\epsilon_{1}+n \hbar \omega$ emerge as well. Now the multiple-photon processes become important, leading to many sideband peaks. The sideband peaks from the ground state are almost symmetric [see Fig. 5(b)], but the sideband peaks of the excited state are significantly asymmetric [see Fig. 5(c)]. 


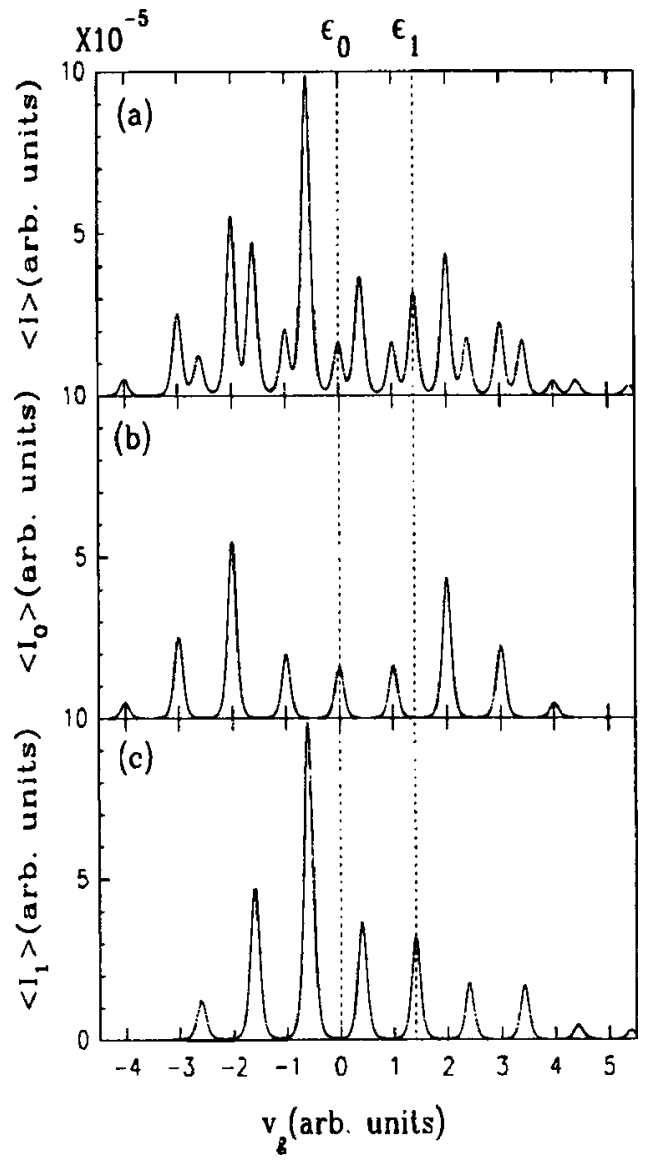

FIG. 5. (a) $\langle I\rangle$ vs $v_{g}$ for $\hbar \omega<\Delta \epsilon$. (b) $\left\langle I_{0}\right\rangle$ vs $v_{g}$. (c) $\left\langle I_{1}\right\rangle$ vs $v_{g}$. $\Delta_{0}=0, \Delta_{L}=\Delta_{R}=3.1, \quad \omega=1, \quad \Gamma_{0}=0.01, \quad \Gamma_{1}=0.03, \Delta \epsilon=1.4, \quad V$ $=0.02$, and $\mathcal{T}=0.05$.

\section{THE DEPENDENCE OF THE INTEGRATION $\int\left\langle I\left(v_{g}\right)\right\rangle d v_{g}$ ON THE INTENSITY OF MW FIELDS}

If the electron-electron Coulomb interaction is neglected $(U=0)$, the integration $\int\langle I\rangle d v_{g}$ can be calculated easily. From Eq. (21), one obtains

$$
G_{j j}^{r}\left(t, t^{\prime}\right)=g_{\epsilon_{j}}^{r}\left(t, t^{\prime}\right) \exp \left\{-\frac{\Gamma_{j}}{2}\left(t-t^{\prime}\right)\right\},
$$

and $\left\langle A_{j}^{\alpha}(\epsilon, t)\right\rangle$ reduces to

$$
\left\langle A_{j}^{\alpha}(\epsilon, t)\right\rangle=\sum_{k} J_{k}^{2}\left(\frac{\Delta_{0}-\Delta_{\alpha}}{\omega}\right) \frac{1}{\epsilon-\epsilon_{j}-k \omega+i \Gamma_{j} / 2} .
$$

Substituting Eq. (31) into Eq. (10), the average current $\langle I\rangle$ is obtained immediately,

$$
\begin{aligned}
\langle I\rangle= & 2 e \sum_{j} \frac{\Gamma_{j}^{L} \Gamma_{j}^{R}}{\Gamma_{j}} \sum_{k} \int \frac{d \epsilon}{2 \pi}\left[f_{L}(\epsilon) J_{k}^{2}\left(\frac{\Delta_{0}-\Delta_{L}}{\omega}\right)\right. \\
& \left.-f_{R}(\epsilon) J_{k}^{2}\left(\frac{\Delta_{0}-\Delta_{R}}{\omega}\right)\right] \times \frac{\Gamma_{j} / 2}{\left(\epsilon-\epsilon_{j}-k \omega\right)^{2}+\Gamma_{j}^{2} / 4} .
\end{aligned}
$$

Then the integration $\int\langle I\rangle d v_{g}$ can be carried out,

$$
\begin{aligned}
\int\left\langle I\left(v_{g}\right)\right\rangle d v_{g} & =\sum_{j} \int\left\langle I_{j}\left(\epsilon_{j}\right)\right\rangle d \epsilon_{j} \\
& =e \frac{\Gamma_{j}^{L} \Gamma_{j}^{R}}{\Gamma_{j}} \int d \epsilon\left[f_{L}(\epsilon)-f_{R}(\boldsymbol{\epsilon})\right] \\
& =e\left(\mu_{L}-\mu_{R}\right) \sum_{j} \Gamma_{j}^{L} \Gamma_{j}^{R} / \Gamma_{j} .
\end{aligned}
$$

Obviously, the integration $\int\left\langle I\left(v_{g}\right)\right\rangle d v_{g}$ is a constant, independent of the intensity of MW fields (the symmetric or asymmetric case) and the temperature $\mathcal{T}$, as long as the Coulomb interaction is neglected.

However, in many experiments the integration $\int\langle I\rangle d v_{g}$ obviously increases with the intensity of MW fields. ${ }^{15,21-24}$ In fact, the electron-electron Coulomb interaction and the many-body effect play important role. With the $e-e$ interaction, although the integration $\int\langle I\rangle d v_{g}$ cannot be performed analytically due to the fact that $n_{j}$ in the expression of $\langle I\rangle$ depends on $v_{g}$; but from the numerical studies mentioned above one easily finds that the integration $\int\langle I\rangle d v_{g}$ increases with the intensity of MW fields due to the participation of the excited state. [See Figs. 1(a), 3(a), and 4(a). For Fig. 5 this property is also satisfied, although the curve without MW fields is not shown there.]

\section{CONCLUSIONS}

In this paper, we have studied the electron tunneling through an ultrasmall quantum dot under the influence of MW fields. Two single electronic states and the intradot Coulomb interaction are considered. By using the nonequilibrium Green function, the time-dependent current $I_{L}(t)$, and the average current $\langle I\rangle$ are derived. The excited-state resonance and its sideband resonances are induced notably for the cases of $\hbar \omega>\Delta \epsilon$ with any intensity of MW fields and $\hbar \omega<\Delta \epsilon$ with the strong intensity of MW fields. The sideband peaks of the ground state are almost symmetric, whereas the sideband peaks of the excited state are heavily asymmetric. Under slightly asymmetric MW fields with suitable magnitudes the sideband peak at $\epsilon_{0}+\hbar \omega$ will disappear. In addition, we found that the integrated current $\left.\int\left\langle I v_{g}\right)\right\rangle d v_{g}$ increases with the intensity of MW fields, which can be attributed to the intradot Coulomb interaction.

\section{ACKNOWLEDGMENTS}

We gratefully acknowledge the financial support by a RGC grant from the SAR Government of Hong Kong under Grant No. HKU 7112/97P, a grant from the Croucher Foundation, a CRCG grant from the University of Hong Kong, and a grant from the Chinese National Natural Science Foundation. We also thank the Computer Center of the University of Hong Kong for computational facilities. Q.F.S. and T.H.L. would like to thank B. G. Wang, Q. R. Zheng, X. A. Zhao, and Y. D. Wei for many helpful discussions. 
*Permanent address: Department of Physics, Peking University, Beijing 100871, China.

${ }^{1}$ P. K. Tien and J. P. Gordon, Phys. Rev. 129, 647 (1963).

${ }^{2}$ A. H. Dayem and R. J. Martin, Phys. Rev. Lett. 8, 246 (1962).

${ }^{3}$ N. S. Wingreen, A. P. Jauho, and Y. Meir, Phys. Rev. B 48, 8487 (1993).

${ }^{4}$ A. P. Jauho, N. S. Wingreen, and Y. Meir, Phys. Rev. B 50, 5528 (1994).

${ }^{5}$ J. Iñarrea, G. Platero, and C. Tejedor, Phys. Rev. B 50, 4581 (1994).

${ }^{6}$ J. Inarrea and G. Platero, Phys. Rev. B 51, 5244 (1995).

${ }^{7}$ K. Yakubo, Shechao Feng, and Qing Hu, Phys. Rev. B 54, 7987 (1996).

${ }^{8}$ C. Bruder and H. Schoeller, Phys. Rev. Lett. 72, 1076 (1994).

${ }^{9}$ T. H. Oosterkamp, L. P. Kouwenhoven, A. E. A. Koolen, N. C. van der Vaart, and C. J. P. M. Harmans, Semicond. Sci. Technol. 11, 1512 (1996).

${ }^{10}$ D. Sokolovski, Phys. Rev. B 37, 4201 (1998).

${ }^{11}$ M. Wagner, Phys. Rev. Lett. 76, 4010 (1996); Phys. Rev. B 49, 16544 (1994).

${ }^{12}$ Q.-F. Sun, J. Wang, and T.-H. Lin, Phys. Rev. B 58, 2008 (1998).

${ }^{13}$ C. A. Stafford and Ned S. Wingreen, Phys. Rev. Lett. 76, 1916 (1996).

${ }^{14}$ Qing-feng Sun and Tsung-han Lin, J. Phys.: Condens. Matter 9, 4875 (1994).

${ }^{15}$ L. P. Kouwenhoven, S. Jauhar, K. McCormick, D. Dixon, P. L. McEuen, Yu. V. Nazarov, N. C. van der Vaart, and C. T. Foxon, Phys. Rev. B 50, 2019 (1994).

${ }^{16}$ P. Johansson and G. Wendin, Phys. Rev. B 46, 1451 (1992).

${ }^{17}$ H. M. Pastawski, Phys. Rev. B 46, 4053 (1992).
${ }^{18}$ M. H. Pedersen and M. Büttiker, cond-mat/9803306.

${ }^{19}$ B. J. Keay, S. J. Allen, Jr., J. Galan, J. P. Kaminski, K. L. Campman, A. C. Gossard, U. Bhattacharya, and M. J. W. Rodwell, Phys. Rev. Lett. 75, 4098 (1995).

${ }^{20}$ B. J. Keay, S. Zeuner, S. J. Allen, Jr., K. D. Maranowski, A. C. Gossard, U. Bhattacharya, and M. J. W. Rodwell, Phys. Rev. Lett. 75, 4102 (1995).

${ }^{21}$ R. H. Blick, R. J. Haug, D. W. van der Weide, K. von Klitzing, and K. Eberl, Appl. Phys. Lett. 67, 3924 (1995).

${ }^{22}$ H. Drexler, J. S. Scott, S. J. Allen, K. L. Campman, and A. C. Gossard, Appl. Phys. Lett. 67, 2816 (1995).

${ }^{23}$ L. P. Kouwenhoven, S. Jauhar, J. Orenstein, P. L. McEuen, Y. Nagamune, J. Motohisa, and H. Sakaki, Phys. Rev. Lett. 73, 3443 (1994).

${ }^{24}$ T. H. Oosterkamp, L. P. Kouwenhoven, A. E. A. Koolen, N. C. van der Vaart, and C. J. P. M. Harmans, Phys. Rev. Lett. 78, 1536 (1997).

${ }^{25} \mathrm{Ph}$. Brune, C. Bruder, and H. Schoeller, Phys. Rev. B 56, 4730 (1997).

${ }^{26}$ N. S. Wingreen, K. W. Jacobsen, J. W. Wilkins, Phys. Rev. B 40, 11834 (1989).

${ }^{27}$ Y. Meir, N. S. Wingreen, and P. A. Lee, Phys. Rev. Lett. 66, 3048 (1991).

${ }^{28}$ For discussions of gauge invariance, see M. Büttiker and T. Christen, in Mesoscopic Electron Transport, Vol. 345 of NATO Advanced Study Institute, Series E: Applied Science, edited by L. L. Sohn, L. P. Kouwenhoven, and G. Schoen (Klumer Academic Publishers, Dordrecht, 1997), p. 259; Z. S. Ma, J. Wang, and H. Guo, Phys. Rev. B 57, 9180 (1998).

${ }^{29}$ Qing-feng Sun and Tsung-han Lin, Phys. Rev. B 56, 3591 (1997). 